\title{
Association study in Mexican patients with thyrotoxic hypokalemic periodic paralysis
}

\author{
MARIO ARTURO BAUTISTA-MEDINA ${ }^{1}$, HUGO LEONID GALLARDO-BLANCO ${ }^{2}$, \\ LAURA ELIA MARTINEZ-GARZA ${ }^{2}$, RICARDO MARTIN CERDA-FLORES ${ }^{3}$, \\ FERNANDO JAVIER LAVALLE-GONZALEZ ${ }^{1}$ and JESUS ZACARIAS VILLARREAL-PEREZ ${ }^{1}$ \\ ${ }^{1}$ Endocrinology Department; ${ }^{2}$ Department of Genetics, University Hospital José Eleuterio González; \\ ${ }^{3}$ School of Nursing, Autonomous University of Nuevo León, Monterrey, Nuevo León 64460, Mexico
}

Received October 22, 2018; Accepted March 26, 2020

DOI: $10.3892 /$ br.2020.1331

\begin{abstract}
Hypokalemic periodic paralysis type 1 (OMIM; HOKPP1) and type 2 (OMIM; HOKPP2) are diseases of the muscle characterized by episodes of painless muscle weakness, and is associated with low potassium blood levels. Hyperthyroidism has been associated with thyrotoxic periodic paralysis (TTPP) (OMIM; TTPP1 and TTPP2), and genetic susceptibility has been implicated. In the present study, the clinical and epidemiological characteristics of patients with TTPP are described, together with their association with
\end{abstract}

Correspondence to: Dr Jesus Zacarias Villarreal-Perez, Endocrinology Department, University Hospital José Eleuterio González, Autonomous University of Nuevo León, Francisco I. Madero Avenue and José Eleuterio González Avenue, Colonia Mitras Centro, Monterrey, Nuevo León 64460, Mexico

E-mail: jesus.villarrealp@uanl.mx

Dr Hugo Leonid Gallardo-Blanco, Department of Genetics, University Hospital José Eleuterio González, Autonomous University of Nuevo León, Francisco I. Madero Avenue and José Eleuterio González Avenue, Colonia Mitras Centro, Monterrey, Nuevo León 64460, Mexico

E-mail: hugo.gallardobl@uanl.edu.mx

Abbreviations: AIM, ancestry informative marker; AFR, African [1,000 genomes project super population]; AMR, ad mixed American [1,000 genomes project super population]; CACNA1s, voltage-gated calcium channel $\alpha 1$ subunit S gene; CLM, Colombians from Medellin, Colombia [1,000 genomes project population]; EAS, East Asian [1,000 genomes project super population]; EUR, European [1,000 genomes project super population]; HOKPP1, hypokalemic periodic paralysis type 1; HOKPP2, hypokalemic periodic paralysis type 2 ; KCNE3, voltage-gated potassium channel subfamily E regulatory subunit 3 gene; MXL, Mexican ancestry from Los Angeles USA [1,000 genomes project population]; MUC1, mucin 1, cell surface associated; SCN4A, voltage-gated sodium channel a subunit 4 gene; SAS, South Asian [1000 genomes project super population]; TTPP, thyrotoxic periodic paralysis

Key words: hypokalemic periodic paralysis, thyrotoxic hypokalemic periodic paralysis, $M U C l$, association study, genetic variant, CACNA1s, KCNE3, SCN4A genetic variants reported previously in other populations. A prospective and a retrospective search of the medical records of patients who attended the emergency department at the Hospital Universitario 'Dr. Jose E. Gonzalez' in Monterrey, Nuevo León, Mexico, and were diagnosed with TTPP was performed. A total of 16 gene variants in the genes $M U C 1$, $C A C N A 1 S, K C N E 3$ and $S C N 4 A$, and nine ancestry informative markers (AIMs), were analysed by Multiplex TaqMan $^{\mathrm{TM}}$ Open Array assay, and a genetic association study was performed. A total of 11 patients were recruited, comprising nine males and two females (age range, 19-52 years) and 64 control subjects. Only two cases (18\%) had a previous diagnosis of hyperthyroidism; the rest were diagnosed subsequently with Graves' disease. Based on the analysis, two DNA variants were found to potentially confer an increased risk for TTPP: SIPRI rs3737576 [odds ratio (OR), 4.38; 95\% confidence interval (CI), 1.08-17.76] and AIM rs2330442 (OR, 4.50; 95\% CI, 1.21-16.69), and one variant was suggested to be possibly associated with TTPP, namely MUC1 rs4072037 (OR, 3.08; 95\% CI, 0.841-1.38). However, there were no statistically significant associations between any of the 24 DNA variants and TTPP in a population from northeast Mexico.

\section{Introduction}

Periodic paralysis is a characteristic of neuromuscular diseases of paroxysmal muscle weakness of the limbs. Common causes of this condition are thyrotoxic periodic paralysis types 1 and 2 (TTPP1 and TTPP2 OMIM \#188580 and \#613239, respectively) (1), which are considered a type of endocrine channelopathy. The acute presentation of flaccid muscular palsy associated with any cause of thyrotoxicosis that produces hypokalaemia is the primary characteristic of TTPP (2). Although TTPP is more common in Asian populations, a significant increase in the number of cases worldwide has been reported, and even though the incidence of hyperthyroidism is higher in women, TTPP is more prevalent in men $(3,4)$. The first clinical manifestation consists of recurrent episodes of muscle weakness, which can vary from a low grade to the most severe forms, with complete flaccid palsy (4). Proximal muscles of the lower limbs are more prone to be associated with paralysis, with no sensitive involvement. Precipitating 
factors for acute episodes are high consumption of simple carbohydrates, alcohol intake and intense exercise (4). Thyroid function testing is a valuable tool to distinguish TTPP (4). In the majority of the cases of TTPP, hyperthyroidism is caused by Graves' disease, which is also the leading cause of hyperthyroidism worldwide (4). However, TTPP may also be associated with other causes of hyperthyroidism, including functional thyroid nodules, toxic multinodular goitre and factitious thyrotoxicosis (5). Gene variants have been reported to be associated with thyrotoxic hypokalemic periodic paralysis (THPP1) [voltage-gated calcium channel $\alpha 1$ subunit $\mathrm{S}$ (CACNA1s) gene], THPP2 [voltage-gated potassium channel subfamily J member 18 (KCNJ18) gene], hypokalemic periodic paralysis type 1 (HOKPP1; CACNA1S gene), and HOKPP type 2 (HOKPP2) [voltage-gated sodium channel a subunit 4 (SCN4A) gene] (1). The voltage-gated calcium channel $\alpha 1 \mathrm{C}$ subunit $(C A C N A 1 C)$ gene is associated with HOKPP1 (OMIM, \#170400) and TTPP1 (OMIM, \#188580) (1), and has been studied in patients with TTPP (1). Variants in other genes that encode for muscle ion channels have also been investigated. In a patient with TTPP of Portuguese descent, an $\mathrm{R} 83 \mathrm{H}$ variant in the voltage-gated potassium channel subfamily E regulatory subunit 3 gene (KCNE3) (6). Since TTPP patients have an increased level of ATPase activity, genes that encode $\mathrm{a}$ and $\mathrm{b}$ adrenergic receptors have been analysed, although no associations have been identified in these cases (6-9). Another study showed that 10 out of 30 Caucasian or Brazilian patients with TTPP possessed a variant in the KCNJ18 gene (associated with TTPP2), an inwardly rectifying potassium channel expressed in skeletal muscles that is transcriptionally regulated by thyroid hormone (10).

Although other ethnicities are affected, TTPP is most prevalent in individuals of Asian and, secondly, of Latin American descendant (4). To the best of our knowledge, there are no previous studies that have been performed on Mexican patients. The aim of the present study was to describe the clinical and epidemiological characteristics of patients with TTPP. An association study was performed with variants in the CACNA1s, mucin 1 (MUC1), KCNE3 and SCN4A genes, as well as in ancestry informative markers (AIMs) and Sphingosine1 Phosphate Receptor 1 (S1PR1) ancestry genes, in a population from northeast Mexico. In total, 9 AIMs were included to analyse population structure and identify outlier subjects, The use of AIMs in population studies can identify population heterogeneity in complex populations and may reduce type I and type II errors in multifactorial diseases and multifactorial traits $(11,12)$.

\section{Materials and methods}

Ethics. The present study was performed at the Genetics and Endocrinology Departments of the University Hospital 'Dr José Eleuterio González' Universidad Autónoma de Nuevo León (UANL) (Monterrey, México), with the approval of the Ethics Committee (approval no. EN13004), University Hospital ‘Dr José Eleuterio González', UANL.

Design of study and subjects. Between May 2012 and December 2014, the prospective recruitment of north-eastern Mexican patients newly diagnosed with TTPP, admitted through the emergency department at the Hospital Universitario
'Dr Jose E. Gonzalez' in Monterrey, Nuevo León, Mexico was performed, as well as a retrospective search of medical records of patients whom had previously been diagnosed with TTPP and had attended the endocrinology outpatient clinic. All subjects accepted and signed informed consent forms. Northeastern Mexican patients, of either sex and all ages were invited to participate in the present study. A total of 11 patients were recruited ( 9 males and two females; age range, 19-52 years; median, 31 years). A clinical evaluation was performed for each patient, which included medical history, thyroid function test, assessment of the blood electrolyte levels, and the type of treatment received. A venous blood sample was obtained and placed in an EDTA vial for DNA extraction. A total of 64 Northeastern Mexican control subjects, including 26 females and 38 males (age range, 19-52 years; median, 41.4 years) were obtained from the DNA bank of the Genetics department at the University Hospital 'Dr. José Eleuterio González’ $(11,12)$.

Laboratory tests. The thyroid profile [thyroid-stimulating hormone (TSH), free thyroxine (FT4), total triiodothyronine (T3T) and total thyroxine (T4T)] were determined by electrochemiluminescence using a Cobas e411 Analyzer (Roche Diagnostics) and T3 uptake was assessed by chemiluminescence (using an IMMULITE 1000 Immunoassay system; MyIMSA Solutions, Ltd.; Siemens Healthineers). The levels of serum and urinary electrolytes were measured using an ion-selective method (Dx 800 Beckman Coulter Hematology Analyzer; Beckman Coulter, Inc.). Blood and urinary samples were taken as routine at the University Hospital 'Dr. José Eleuterio González', and assessed according to the manufacturer's protocol.

DNA extraction. DNA extraction was performed using $200 \mu \mathrm{l}$ EDTA treated whole blood samples, using a QIAamp ${ }^{\circledR}$ Blood Mini kit on the automated QIAcube system (Qiagen $\mathrm{GmbH}$ ). Purified DNA was collected, diluted to a concentration of $50 \mathrm{ng} / \mu \mathrm{l}$ [determined by measuring the absorbance (A) at $\mathrm{A}_{260 / 280}$ and $\mathrm{A}_{260 / 230}$ to be 1.6 to 1.9 ], and stored at $-20^{\circ} \mathrm{C}$ until further use $(11,12)$.

Open array reactions. Open array was performed using the TaqMan ${ }^{\circledR}$ assay (Applied Biosystems; Thermo Fisher Scientific, Inc.), and genotypes were captured using the OpenArray ${ }^{\circledR}$ NT Genotyping system (Applied Biosystems; Thermo Fisher Scientific, Inc.). Non-template control (process control) was used in each plate assay. DNA samples (50 ng/ $\mu$ l each sample) and TaqMan ${ }^{\circledR}$ OpenArray Master mix (Applied Biosystems; Thermo Fisher Scientific, Inc.) were mixed in a 384 well plate and transferred to the OpenArray plate using an autoloader. The OpenArray plate was subsequently filled with immersion fluid, and sealed with glue. Multiplex TaqMan ${ }^{\circledR}$ assay reactions were performed in a Dual Flat Block GeneAmp PCR system 9700 (Applied Biosystems; Thermo Fisher Scientific, Inc.). The PCR cycling conditions were as follows: Initial denaturation at $93^{\circ} \mathrm{C}$ for $10 \mathrm{~min}$; followed by 50 cycles at $95^{\circ} \mathrm{C}$ for $45 \mathrm{sec}$, $94^{\circ} \mathrm{C}$ for $13 \mathrm{sec}$ and $53^{\circ} \mathrm{C}$ for $14 \mathrm{sec}$; and a final step at $25^{\circ} \mathrm{C}$ for $2 \mathrm{~min}$. Samples were stored at $4^{\circ} \mathrm{C}$ until further use $(11,12)$.

Genotype analysis. Genotype analysis was analysed using the TaqMan ${ }^{\circledR}$ Genotyper Software version 1.0, according to 
the manufacturer's protocol (Applied Biosystems; Thermo Fisher Scientific, Inc.). The accuracy of the genotyping was assessed by comparison with 15 samples, all of which had been genotyped three times, resulting in 45 comparisons per single nucleotide polymorphism (SNP).

Genotype association test. Genetic data were analysed using the Golden Helix SNP \& Variation Suite version 8.7 program (Golden Helix, Inc.), with the Homo sapiens GRCh37/hg19 genome assembly. The 24 SNPs were analysed to detect possible deviations from the Hardy-Weinberg equilibrium (HWE) using a Fisher's exact test $(\mathrm{P}<0.01$ was considered to indicate a statistically significant difference) (11). Association studies were performed using the dominant genetic model. Fisher's exact test was used to estimate P-values, odds ratios (ORs), and 95\% confidence intervals (CIs). Fisher's exact test P-values, corrected using the Bonferroni method, and false discovery rates (FDRs) were calculated to exclude spurious associations. $\mathrm{P}<0.05$, corrected by Bonferroni's method and FDR, was considered to indicate a statistically significant difference (11).

\section{Results}

Clinical characteristics. A total of 11 northeastern Mexican patients with THPP were evaluated. Hypokalaemia was detected at the onset of paralysis symptoms. Of the 11 patients, 10 were recruited during the acute onset of symptoms, and a further patient through a retrospective search of cases. The majority of the cases were male (82\%). Hypokalaemia was associated with the severity of the clinical manifestations, which varied from muscle weakness to muscular palsy. No family cause of hyperthyroidism was identified, instead all cases were sporadic cases. Table I shows the laboratory findings, including the low urinary and serum potassium values (range: $15-30 \mathrm{mM} / \mathrm{l}$, and $1.2-2.4, \mathrm{mM} / \mathrm{l}$ ). The thyroid hormones profile revealed a $\mathrm{TSH} \leq 0.01$ in all patients, with significant increases observed for T3 Cap, T3T, FT4 and T4T. Of these, 2 patients disclosed a precipitant factor in the hours prior to the event, one patient had had a meal abundant in carbohydrates, and the other patient had engaged in vigorous physical activity. Additionally, 4 patients had a history of similar episodes, and received treatment with potassium replacement in other hospitals, where the cause of the hypokalaemia was not determined, entailing further hypokalemic crisis. The remaining 5 patients developed hyperkalaemia as a side effect of routinely receiving potassium replacement. After the diagnosis of Graves' disease as the cause of the hypokalaemia, the 11 patients received ${ }^{131}$ I-iodine, which halted the hypokalemic crisis (Table I).

Genotype analysis. The allelic and genotype frequencies, and HWE P-values calculated for the 24 SNPs, in cases and in control subjects, and are presented in Table II. These results show that all the control subjects and patients were in HWE ( $P>0.01)$. The associations of the 24 gene variants were analysed; 15 variants were from genes associated with electrolyte membrane transport, and had been previously reported to be associated with TTPP in other populations: CACNA1S (13); rs12139527, rs1546416, rs3767500, rs3818873, rs1998721, rs4915212, rs9427714, rs734881, rs4951629, rs12561765 and rs2296383, KCNE3 (6); rs17215437 and SCN4A (14,15); and

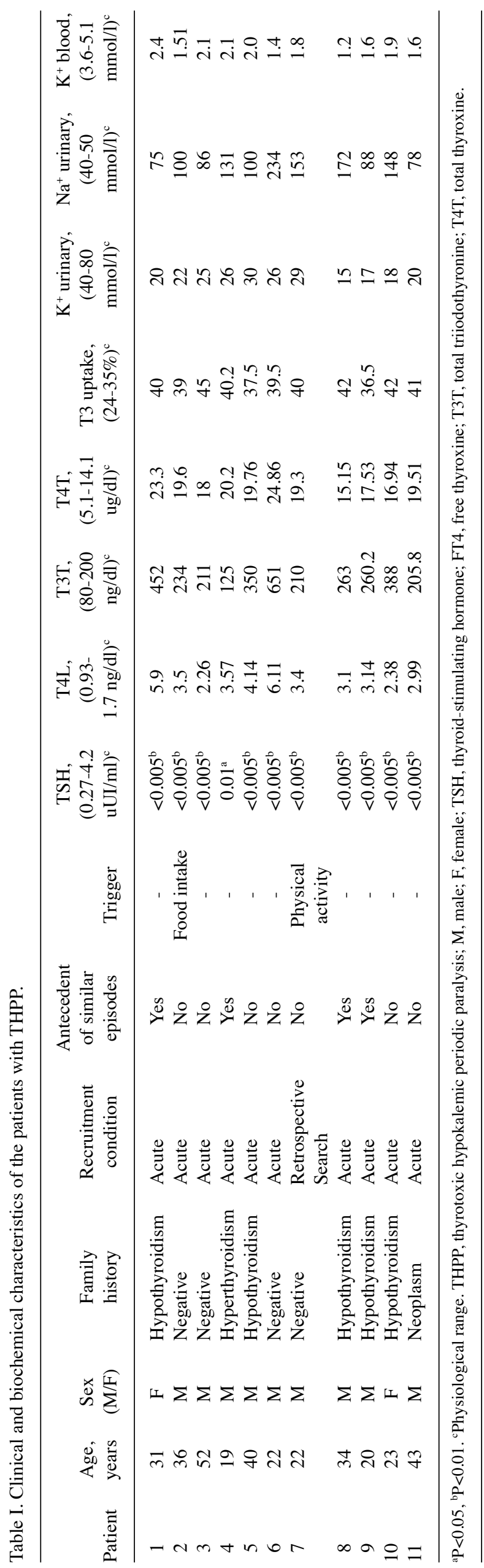


rs2070720, rs2058194 and rs9894841. Additionally, 9 AIM variants: S1PR1 (rs3737576), ATF3 (rs4951629), phospholipase 5 (PLD5; rs316873), elastin (ELN; rs868005 and rs2239691), lysine demethylase 2A (KDM2A; rs11227699), AMZ2P1; GNA13 (rs11652805, rs2330442 and rs10954737); and the MUC1 gene (rs4072037) were also included (16).

Genotype association test. Based on the 24 SNPs, only two genetic variants were predicted to confer a risk for TTPP with a significant OR (Table II): S1PRI rs3737576 (T allele; OR, 4.38; 95\% CI, 1.08-17.76, C allele; OR, 0.23; 95\% CI, 0.06-0.92), and the AIM rs2330442 (OR, 4.50; 95\% CI, 1.21-16.69, A allele; OR, 0.22; 95\% CI, 0.06-0.82, G allele). The variant $M U C 1$ rs4072037 (OR, 3.08; 95\% CI, 0.84-11.38) could confer risk to TTPP. After applying the Bonferroni and FDR corrections to the P-values, statistical significance was no longer detected.

\section{Discussion}

TTPP is a rare disease, its prevalence among patients with thyrotoxicosis in the US is estimated to be between $0.1-0.2 \%$, and the incidence in Japanese and other Asian populations has been declining in the last 10 years (17). The majority of the information regarding the pathology of TTPP has been obtained through case reports, predominantly from Asian patients (17). Due to the number of TTPP patients diagnosed in the Hospital Universitario 'Dr Jose E. Gonzalez' between May 2013 and December 2014, the present study aimed to determine common precipitating risk factors and to identify genetic variants associated with this disorder in a northeastern Mexican population. All patients had symptoms of severe hypokalaemia and flaccid muscle palsy, particularly of the lower limbs at their admission to the emergency department. Hypokalaemia was caused by a massive shift in potassium concentration from extracellular to intracellular space. Increased excretion is the most common mechanism underlying hypokalaemia, and thus it should be determined whether potassium replacement may exacerbate the condition, causing arrhythmias or even death, following correction of hypokalaemia (18). A careful clinical evaluation of patients with hypokalaemia should be made to eliminate the possibility of thyrotoxicosis as the cause of the electrolyte alterations.

Genetic predisposition in hypokalemic periodic paralysis has been investigated, and several gene variants have been reported to be associated with TTPP. Gene variants in genes associated with ion channel transport are predominantly associated with TTPP, such as the CACNAIC gene, which is involved calcium transport and is associated with TTPP in the Chinese population $(14,19)$. Furthermore, variants in genes associated with potassium channels, such as the KCNE3 gene that encodes muscle ion channels, have been identified in a patient of Portuguese descent (6), and the KCNJ18 (Kir2.6) gene has been identified in Brazilian patients (10). In the present study; however, no such associations were identified between these genes and TTPP in our population.

Previously, it has been shown that $M U C 1$ regulates the renal calcium channel TRPV5, and has a putative protective role against calcium nephrolithiasis via increasing urinary calcium reabsorption (20). Homeostasis of calcium and potassium are connected; therefore, this may underlie the interaction and association between genetic variants of $M U C 1$ with hypokalaemia. The C allele in rs4072037 of the $M U C 1$ gene is associated with a decrease in magnesium levels $(21,22)$. A previous study reported that hypomagnesemia is associated with hypokalaemia, which is mediated by stimulation of the renal outer medullary potassium channels, resulting in increased potassium excretion (23). Previously published clinically relevant data concerning the association between potassium and magnesium showed that $>50 \%$ of cases of clinically significant hypokalaemia is accompanied by concomitant magnesium deficiency, and is clinically most frequently observed in individuals receiving loop or thiazide diuretic therapy. Concomitant magnesium deficiency has long been appreciated to aggravate hypokalaemia. Hypokalaemia associated with magnesium deficiency is often refractory to treatment with potassium (24).

The T allele (rs3737576) of the S1PRl gene had a lower frequency in Latino American populations (AMR), and has a higher frequency in Mexicans from Los Angeles (MXL) and Peruvians of Lima Peru (PEL) when compared with other worldwide populations (Jan2020.archive.ensembl. org/Homo_sapiens/Variation/Population?db=core;r=1:101243 507-101244507; $\mathrm{v}=\mathrm{rs} 3737576 ; \mathrm{vdb}=$ variation; $\mathrm{vf}=2090687)(25)$. The genotype TT (rs3737576) was identified more frequently in the cases of the AMR and MXL populations when compared with controls, which suggested that ancestry may be a relevant component in assessing the risk of TTPP. Thus, TTPP replication association studies in AMR populations are required to corroborate the preliminary findings of this pilot study. The S1PRl gene has been shown to inhibit kidney epithelial-mesenchymal transition initiated by ischemia/reperfusion injury via the phosphoinositide 3 kinase/Akt pathway, and reperfusion can result in hyperkalaemia (26).

AIM rs2330442 is a human-specific segregation variant (16) (A low-risk allele, and $G$ risk allele, in the present study). Allele A was found to have a lower frequency in cases and in the AFR super population when compared with controls, AMR, East Asian (EAS), South Asian (SAS) and EUR super populations and MXL population (Jan2020. archive.ensembl.org/Homo_sapiens/Variation/Population?d $\mathrm{b}=$ core; $\mathrm{r}=7: 42339972-42340972 ; \mathrm{v}=\mathrm{rs} 2330442 ; \mathrm{vdb}=$ variatio $\mathrm{n} ; \mathrm{vf}=17272273$ ) (25). TPP occurs more frequently in young Asian and Latin American males compared with Caucasians or Africans $(8,27)$. The rs2330442 variant (Allele A, OR, $4.50 ; 95 \% \mathrm{CI}, 1.21-16.69)$ is located between two enhancer sequences in ENSR00001124514 (inactive in the adrenal gland) and ENSR00001124515 (interesting repressed in the adrenal gland; ENSEMBL; Jan2020.archive.ensembl. org/Homo_sapiens/Location/View?db=core;r=7:42340222 -42340723; $\mathrm{v}=\mathrm{rs} 2330442 ; \mathrm{vdb}=$ variation; $\mathrm{vf}=17272273)$. The rs2330442 variant is included inside as a constrained element (a functional element in a reference genome; a conserved sequence across 100 eutherian mammal species) (28).

The present study is the first to have reported on the potential genetic associations of patients with in a northeastern Mexican cohort, to the best of our knowledge. The allelic frequency of DNA variants with the risk associated with TTPP in cases from northeastern Mexico was assessed; however, none of the variants were significantly associated, and a significant risk with TTPP was not identified. 


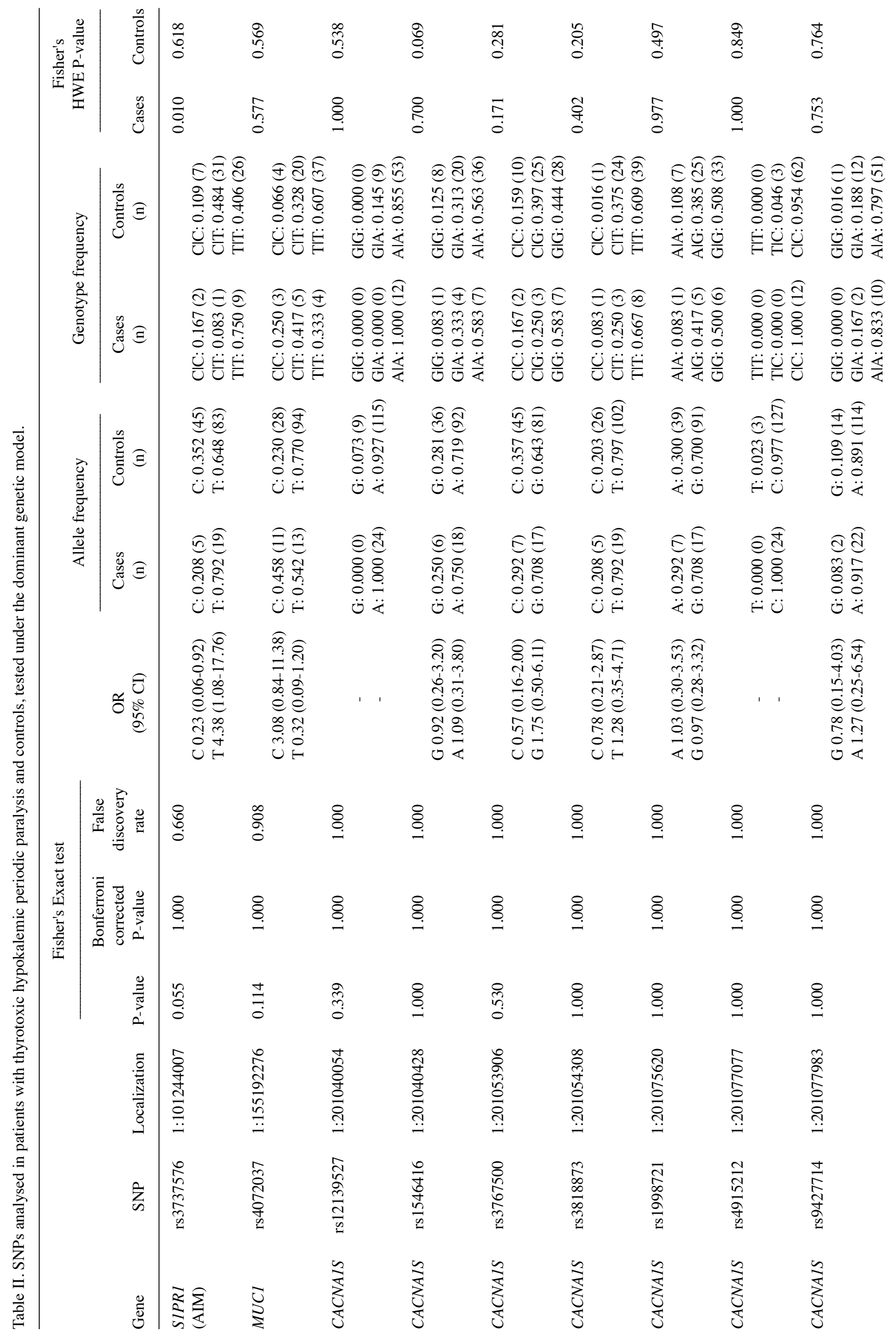




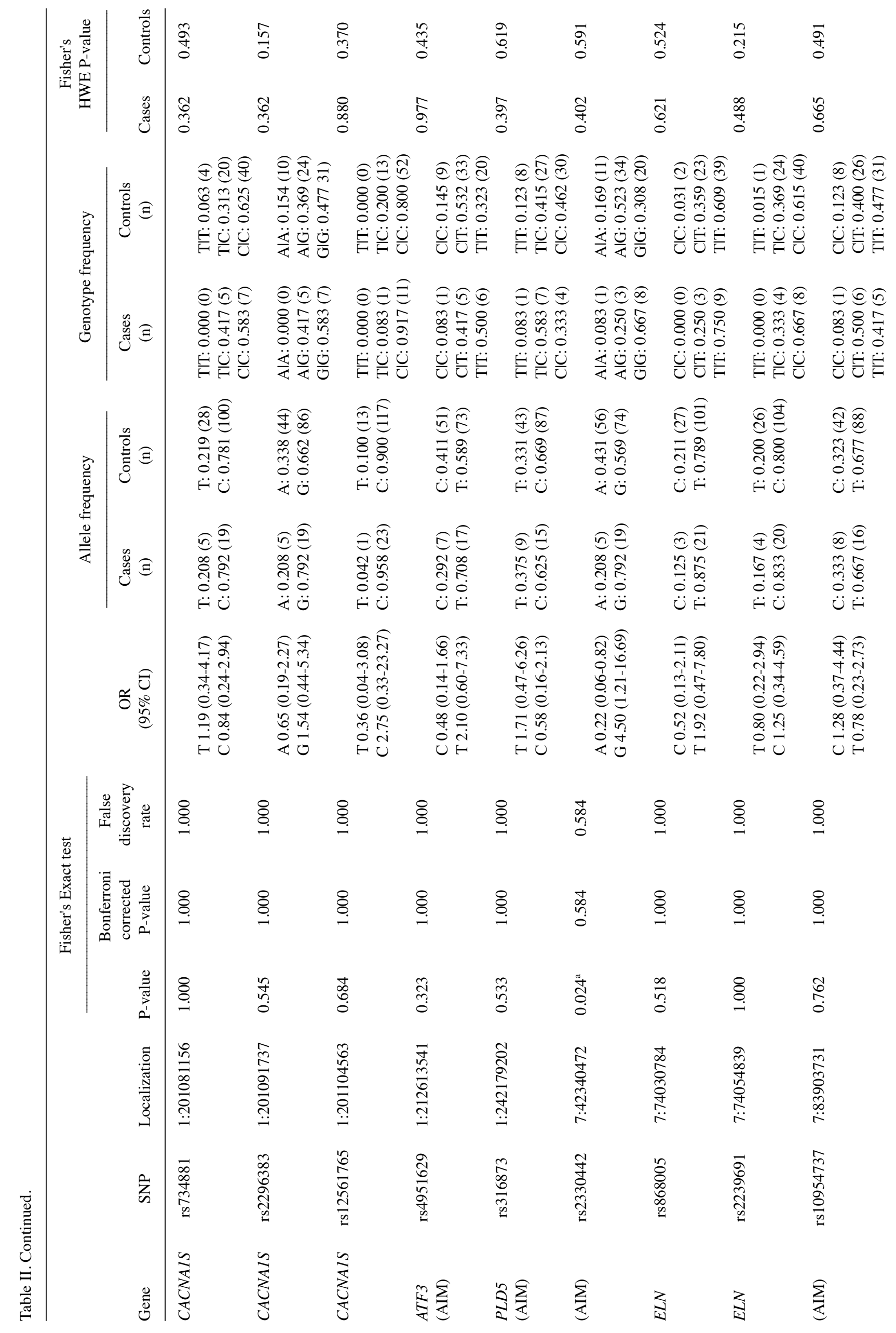




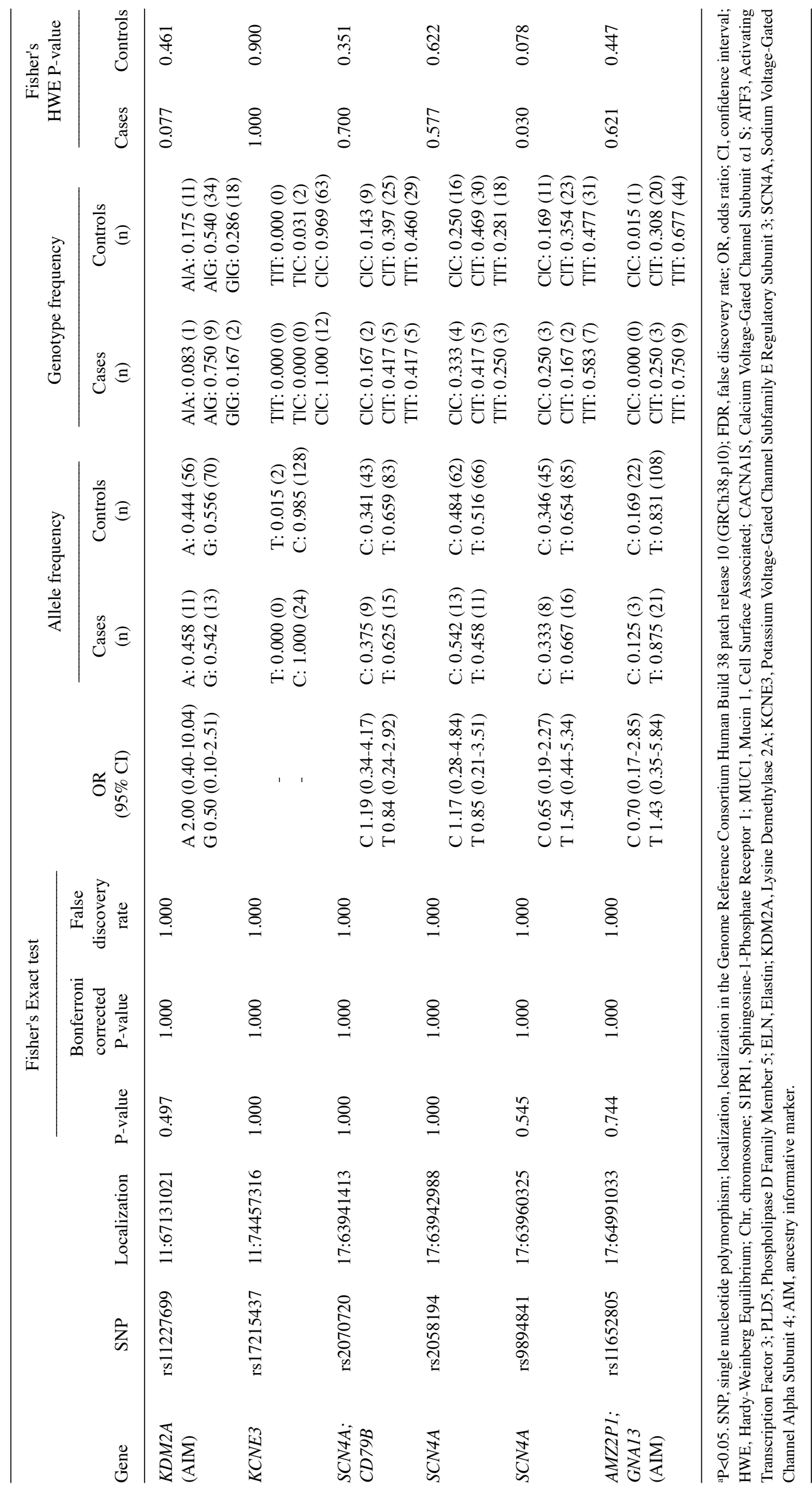


The C allele rs3737576 of the S1PRI gene (chromosome 1) had a lower frequency in the AMR super population when compared with the controls of the present study and AMR super population. The AIM rs2330442 (chromosome 7), allele G has been shown to have a higher frequency in cases and the AFR super population when compared with controls, AMR, SAS, EAS and EUR super populations, and the MXL population (25).

TTPP has been reported to occur with differential frequency in other populations, identified more frequently in male Latino American and Asian populations. The Mexican population is a model for genetic studies due to the great ethnic diversity and complexity within native and Mestizo populations (29). Additional TTPP replication association studies in AMR populations are required to corroborate the preliminary findings in the present pilot study. Full sequencing of the CACNA1s, MUC1, KCNE3 and SCN4A genes would also be advantageous as it may be used to identify novel mutations and variations that are associated with TTPP. Additionally, exome and whole-genome studies may be necessary to investigate the above genes and metabolic pathways involved in magnesium and potassium transport and homeostasis.

\section{Acknowledgements}

The authors would like to thank Dr Andrés Fernández Gancedo, Dr Ana Sofía Ríos, Dr Daniel Eduardo Cordova Galván, Miss Iris Carmen Torres Muñoz, and Dr Fabiola Yazmin Agüero Zapata for assistance in the collection of clinical data and sample handling.

\section{Funding}

The present study was funded by the Endocrinology Department and the Department of Genetics, University Hospital José Eleuterio González (Monterrey, Mexico).

\section{Availability of data and materials}

The datasets used and/or analysed during the present study are available from the corresponding author on reasonable request.

\section{Authors' contributions}

MABM, HLGB, JZVP, RMCF, FJLG and LEMG designed the study. MABM, FJLG and JZVP contributed to the recruitment of the patients and performed the medical examinations. MABM and HLGB performed the bioinformatics, statistical, and genetics analysis. All authors read and approved the final manuscript.

\section{Ethics approval and consent to participate}

The present study was approved by the Ethics Committee of University Hospital 'Dr. José Eleuterio González', UANL (approval no. EN13004). All patients provided written informed consent.

\section{Patient consent for publication}

Not applicable.

\section{Competing interests}

The authors declare that they have no competing interests.

\section{References}

1. Amberger JS, Bocchini CA, Scott AF and Hamosh A: OMIM. org: Leveraging knowledge across phenotype-gene relationships. Nucleic Res 47: D1038-D1043, 2019.

2. Balakrishnan RK, Chandran SR, Thirumalnesan $G$ and Doraisamy N: Thyrotoxic periodic paralysis. Indian J Endocrinol Metabolism 15 (Suppl 2): S147-S149, 2011.

3. Schalin-Jantti C, Laine T, Valli-Jaakola K, Lonnqvist T, Kontula K and Valimaki MJ: Manifestation, management and molecular analysis of candidate genes in two rare cases of thyrotoxic hypokalemic periodic paralysis. Horm Res 63: 139-144, 2005.

4. Kung AW: Clinical review: Thyrotoxic periodic paralysis: A diagnostic challenge. J Clin Endocrinol Metab 91: 2490-2495, 2006.

5. Tran HA: Inadvertent iodine excess causing thyrotoxic hypokalemic periodic paralysis. Arch Intern Med 165: 2536: 2536, 2005.

6. Dias Da Silva MR, Cerutti JM, Arnaldi LA and Maciel RM: A mutation in the KCNE3 potassium channel gene is associated with susceptibility to thyrotoxic hypokalemic periodic paralysis. J Clin Endocrinol Metab 87: 4881-4884, 2002.

7. Kim TY, Song JY, Kim WB and Shong YK: Arg16Gly polymorphism in beta2-adrenergic receptor gene is not associated with thyrotoxic periodic paralysis in Korean male patients with Graves' disease. Clin Endocrinol (Oxf) 62: 585-589, 2005.

8. Kung AW, Lau KS, Cheung WM and Chan V: Thyrotoxic periodic paralysis and polymorphisms of sodium-potassium ATPase genes. Clin Endocrinol (Oxf) 64: 158-161, 2006.

9. Wang W, Jiang L, Ye L, Zhu N, Su T, Guan L, Li X and Ning G: Mutation screening in Chinese hypokalemic periodic paralysis patients. Mol Genet Metab 87: 359-363, 2006.

10. Ryan DP, da Silva MR, Soong TW, Fontaine B, Donaldson MR, Kung AW, Jongjaroenprasert W, Liang MC, Khoo DH, Cheah JS, et al: Mutations in potassium channel Kir2.6 cause susceptibility to thyrotoxic hypokalemic periodic paralysis. Cell 140: 88-98, 2010.

11. Villarreal-Martinez A, Gallardo-Blanco H, Cerda-Flores R, Torres-Muñoz I, Gómez-Flores M, Salas-Alanís J, Ocampo-Candiani J and Martínez-Garza L: Candidate gene polymorphisms and risk of psoriasis: A pilot study. Exp Ther Med 11: 1217-1222, 2016.

12. Gallardo-Blanco HL, Villarreal-Perez JZ, Cerda-Flores RM, Figueroa A, Sanchez-Dominguez CN, Gutierrez-Valverde JM, Torres-Muñoz IC, Lavalle-Gonzalez FJ, Gallegos-Cabriales EC and Martinez-Garza LE: Genetic variants in KCNJ11, TCF7L2 and HNF4A are associated with type 2 diabetes, BMI and dyslipidemia in families of Northeastern Mexico: A pilot study. Exp Ther Med 13: 523-529, 2017.

13. Carpenter D, Ringrose C, Leo V, Morris A, Robinson RL, Halsall PJ, Hopkins PM and Shaw MA: The role of CACNA1S in predisposition to malignant hyperthermia. BMC Med Genet 10: 104, 2009.

14. Kung AW, Lau KS, Fong GC and Chan V: Association of novel single nucleotide polymorphisms in the calcium channel alpha 1 subunit gene $(\mathrm{Ca}(\mathrm{v}) 1.1)$ and thyrotoxic periodic paralysis. J Clin Endocrinol Metab 89: 1340-1345, 2004.

15. Richards S, Aziz N, Bale S, Bick D, Das S, Gastier-Foster J, Grody WW, Hegde M, Lyon E, Spector E, et al: Standards and guidelines for the interpretation of sequence variants: A joint consensus recommendation of the American college of medical genetics and genomics and the association for molecular pathology. Genet Med 17: 405-424, 2015.

16. Kosoy R, NassirR, Tian C, White PA,Butler LM, Silva G,Kittles R, Alarcon-Riquelme ME, Gregersen PK, Belmont JW, et al: Ancestry informative marker sets for determining continental origin and admixture proportions in common populations in America. Hum Mutat 30: 69-78, 2009.

17. Kwon H, Jung JH, Han KD, Park YG, Cho JH, Lee DY, Han JM, Park SE, Rhee EJ and Lee WY: Prevalence and annual incidence of thyroid disease in Korea from 2006 to 2015: A nationwide population-based cohort study. Endocrinol Metab (Seoul) 33: 260-267, 2018. 
18. Lu KC,Hsu YJ, Chiu JS, Hsu YD and Lin SH: Effects of potassium supplementation on the recovery of thyrotoxic periodic paralysis. Am J Emerg Med 22: 544-547, 2004.

19. Dias da Silva MR, Cerutti JM, Tengan CH, Furuzawa GK, Vieira TC, Gabbai AA and Maciel RM: Mutations linked to familial hypokalaemic periodic paralysis in the calcium channel alphal subunit gene (Cav1.1) are not associated with thyrotoxic hypokalaemic periodic paralysis. Clin Endocrinol(Oxf) 56: 367-375, 2002

20. Nie M, Bal MS, Yang Z, Liu J, Rivera C, Wenzel A, Beck BB Sakhaee K, Marciano DK and Wolf MT: Mucin-1 increases renal trpv5 activity in vitro, and urinary level associates with calcium nephrolithiasis in patients. J Am Soc Nephrol 27: 3447-3458, 2016.

21. Meyer TE, Verwoert GC, Hwang SJ, Glazer NL, Smith AV, van Rooij FJ, Ehret GB, Boerwinkle E, Felix JF, Yang Q, et al: Genome-wide association studies of serum magnesium, potassium, and sodium concentrations identify six Loci influencing serum magnesium levels. PLoS Genet 6: e1001045, 2010.

22. Tin A, Kottgen A, Folsom AR, Maruthur NM, Tajuddin SM, Nalls MA, Evans MK, Zonderman AB, Friedrich CA, Boerwinkle E, et al: Genetic loci for serum magnesium among African-Americans and gene-environment interaction at MUC1 and TRPM6 in European-Americans: The Atherosclerosis Risk in Communities (ARIC) study. BMC Genet 16: 56, 2015.

23. Blaine J, Chonchol M and Levi M: Renal control of calcium, phosphate, and magnesium homeostasis. Clin J Am Soc Nephrol 10: 1257-1272, 2015.
24. Kardalas E, Paschou SA, Anagnostis P, Muscogiuri G, Siasos G and Vryonidou A: Hypokalemia: A clinical update. Endocr Connect 7: R135-R146, 2018

25. 1000 Genomes Project Consortium; Auton A, Brooks LD, Durbin RM, Garrison EP, Kang HM, Korbel JO, Marchini JL, McCarthy S, McVean GA and Abecasis GR: A global reference for human genetic variation. Nature 526: 68-74, 2015.

26. Wang W, Wang A, Luo G, Ma F, Wei X and Bi Y: S1P1 receptor inhibits kidney epithelial mesenchymal transition triggered by ischemia/reperfusion injury via the PI3K/Akt pathway. Acta Biochim Biophys Sin (Shanghai) 50: 651-657, 2018.

27. Silva MR, Chiamolera MI, Kasamatsu TS, Cerutti JM and Maciel RM: Thyrotoxic hypokalemic periodic paralysis, an endocrine emergency: Clinical and genetic features in 25 patients. Arq Bras Endocrinol Metabol 48: 196-215, 2004 (In Portuguese).

28. Garber M, Guttman M, Clamp M, Zody MC, Friedman N and Xie X: Identifying novel constrained elements by exploiting biased substitution patterns. Bioinformatics 25: i54-i62, 2009.

29. Moreno-Estrada A, Gignoux CR, Fernández-López JC, Zakharia F, Sikora M, Contreras AV, Acuña-Alonzo V, Sandoval K, Eng C, Romero-Hidalgo S, et al: Human genetics. The genetics of Mexico recapitulates Native American substructure and affects biomedical traits. Science 344: 1280-1285, 2014.

This work is licensed under a Creative Commons

Attribution-NonCommercial-NoDerivatives 4.0 International (CC BY-NC-ND 4.0) License. 